\title{
Breastfeeding duration and previous breastfeeding experience
}

\author{
E Nagy $^{1}$, H Orvos $^{2}$, A Pál $^{2}$, L Kovács ${ }^{2}$ and K Loveland ${ }^{3}$ \\ Institute of Behavioural Sciences ${ }^{1}$, Semmelweis Medical University, Budapest, Hungary; Department of Obstetrics and Gynecology ${ }^{2}$, \\ Albert Szent-Györgyi Medical University, Szeged, Hungary; Center for Human Development Research ${ }^{3}$, Department of Psychiatry and \\ Behavioral Sciences, University of Texas, Houston Medical School, Houston, Texas, USA
}

\begin{abstract}
Nagy E, Orvos H, Pál A, Kovács L, Loveland K. Breastfeeding duration and previous breastfeeding experience. Acta Pædiatr 2001; 90: 51-56. Stockholm. ISSN 0803-5252

The aim of this study was to examine the effect of duration of earlier breastfeeding on the duration of breastfeeding with subsequent births. Medical records of 327 women who had had at least two deliveries were analysed; 89 of these women had had three deliveries. The average duration of breastfeeding increased from 6.01 mo to 6.85 mo with the second baby, but did not increase significantly with the third. A significant negative correlation was found between duration of first breastfeeding and change of breastfeeding duration at the second breastfeeding. Mothers who breastfed their first child for 0-7 mo significantly increased the duration of breastfeeding with the second child, whereas mothers who breastfed their firstborn for 8 mo or longer significantly reduced breastfeeding with the second baby.
\end{abstract}

Conclusion: Results support earlier studies showing that duration of breastfeeding of the second child is significantly related to previous breastfeeding experience. Results also suggest that previous breastfeeding experience may lead mothers to regulate duration of subsequent breastfeeding toward a level that is optimal for them. The results are interpreted with respect to societal changes in breastfeeding patterns, as well as the role of maternal learning in breastfeeding behaviour.

Key words: Breastfeeding, maternal behaviour

Emese Nagy, Institute of Behavioural Sciences, Semmelweis Medical University, HU-1088, 4. Nagyvárad sq., Budapest, Hungary (Tel. +36 121029 53, fax. +36 121029 55, e-mail. nagyeme@net.sote.hu).Present address: Center for Human Development Research, Department of Psychiatry and Behavioral Sciences, University of Texas-Houston Medical School, UT-MSI, 1300 Moursund Street, Houston, TX 77030, USA (Tel. +1 713500 2591, fax. +1 713500 2585; e-mail. Emese.Nagy@uth.tmc.edu)

Maternal nurturing has a strong hormonal and genetic basis; however, social learning also plays an important role. Suomi and Ripp (1) have shown that neglected rhesus monkeys separated from their mothers at an early age are abusive or neglectful of their own firstborn, but that their maternal behaviour improves with the second or thirdborn. Their findings suggest that social learning of maternal skills improves mothering behaviour with later offspring. In human mothers, little is known about the role of social learning through maternal experience in affecting the nature of nurturing behaviour with later births. Breastfeeding is a maternal behaviour that is of central importance to attachment as well as to infant health. It is therefore of interest to know whether the likelihood and the duration of human mothers' breastfeeding may be influenced by prior maternal experience with breastfeeding.

Several studies have taken into account parity as a variable when examining changes in breastfeeding incidence or duration. In a representative sample from the USA, Ryan and Martinez (2) found that parity alone was not predictive of the duration of breastfeeding.
Macaulay et al. (3) found that the incidence of breastfeeding, and Hill et al. (4) the duration of breastfeeding, was not associated with parity in their studies. MacGowan et al. (5) found that, as parity increased, the incidence of breastfeeding increased until a woman had more than three children, but this finding did not reach statistical significance. Taken together, these studies do not show a clear, significant statistical relationship between breastfeeding incidence or duration and parity.

Most studies have emphasized the importance of breastfeeding experience on later breastfeeding rather than the importance of parity itself. In a longitudinal, prospective study in Denmark, Vestermark et al. (6) found that mothers who had breastfed a child usually breastfed the next child for a period of similar length. And in California, Da Vanzo et al. (7) found that women who breastfed their first child were very likely to breastfeed their later child. Becerra et al. (8) found that mothers in Puerto Rico who breastfed their previous child were 7.3 times more likely to breastfeed the next child than were mothers who had not previously 
Table 1. Sample characteristics .

\begin{tabular}{|c|c|c|c|c|c|c|}
\hline \multirow[b]{2}{*}{ Birth } & \multirow{2}{*}{$\begin{array}{l}\text { Age of mother } \\
\text { (years) (SD) }\end{array}$} & \multirow{2}{*}{$\begin{array}{c}\text { Weight / } \\
\text { grams/ (SD) }\end{array}$} & \multirow{2}{*}{$\begin{array}{l}\text { GA /weeks/ } \\
\text { (SD) }\end{array}$} & \multirow{2}{*}{$\begin{array}{c}\text { Breastfeeding } \\
\text { (months) }(\mathrm{SD}) \text { and } \\
\text { range }\end{array}$} & \multicolumn{2}{|c|}{ Gender } \\
\hline & & & & & Male & Female \\
\hline Birth $1(n=327)$ & $21.8(3.5)$ & $3296(452)$ & $39.6(0.9)$ & $6.02(5.77) 0-42$ & 181 & 146 \\
\hline Birth $2(n=327)$ & $25.5(4.5)$ & 3388 (468) & $39.6(0.8)$ & $6.85(6.62) 0-54$ & 163 & 164 \\
\hline Birth $3(n=89)$ & $27.7(4.8)$ & 3354 (508) & $39.5(0.9)$ & $7.16(7.33) 0-36$ & 43 & 46 \\
\hline
\end{tabular}

breastfed. They suggested that this difference could be based on the previous breastfeeding experience, rather than on parity itself.

Similarly, in Sweden Hornell et al. (9) found that the duration of breastfeeding was significantly correlated with the previous breastfeeding experience. Jones et al. (10) showed that mothers breastfed longer at the second and third births than at the first birth. Victora et al. (11) found that in Brazil the duration of breastfeeding for the second child increased directly according to the length of time the previous child had been breastfed, suggesting that breastfeeding experience plays a role in subsequent breastfeeding.

The aim of our study was to examine the possible relationship in Hungary between breastfeeding duration and previous breastfeeding experience, as reflected in previous breastfeeding duration.

\section{Methods}

Retrospective data were obtained from the medical records of women who visited an obstetrics-gynaecology clinic at the Albert Szent-Gyorgyi Medical University, in Szeged, Hungary in a 6 mo period between January and June 1998. Medical histories were taken by the clinic obstetricians, with the same questions given to each woman. From 708 consecutive cases, we selected those who had had their first two to three deliveries between 1980 and 1997, and for whom all deliveries were vaginal, without complications, and with gestational age of all three newborns 38 wk or more. With this selection, our sample consisted of the medical records of 327 women who had had at least two deliveries; 89 of these women had had three deliveries. Time in years between two consecutive deliveries was taken from the medical histories, but infant's year of birth was not. This research was reviewed and approved by the Ethics Board of the Albert Szent-Gyorgyi Medical University.

Age of the mother at delivery in years, weight of the newborn at delivery in grams, gender of the baby, duration of breastfeeding in months, gestational age of the newborn in weeks, and calculated differences between variables (change scores) were all analysed using the SPSS 7.5 statistical program. Wilcoxon paired comparison tests, bivariate correlations, and linear regression analysis were used to analyse the data. An alpha level of 0.05 for statistical significance was accepted throughout.

\section{Results}

Table 1 gives the characteristics of the study sample and the duration of breastfeeding; Table 2 the breastfeeding durations in the sample by percentile.

\section{Duration of breastfeeding and previous breastfeeding experience}

The Wilcoxon test showed that duration of breastfeeding $(Z=-2.46, p=0.014)$ significantly increased in the second-born compared to the first, but did not increase significantly with the third baby (breastfeeding $Z=$ $-1.48, p=0.14)$. The other variable that showed the same pattern of change was weight of the newborn (1st and 2nd baby: $Z=-3.49, p<0.001 ; 2$ nd and 3rd baby: $Z=-0.96$, n.s.).

Using bivariate correlation we examined the relationships among durations of subsequent breastfeedings. Table 3 shows that the duration of all subsequent breastfeedings had significant positive correlations with the duration of previous breastfeeding. However, changes in the duration of breastfeeding (the differences between subsequent breastfeedings in months) showed significant negative correlations with previous breastfeeding duration. That is, the greater the mother's duration of breastfeeding with her baby, the less was the increase in duration with her next baby. In fact, mothers who breastfed for a short time with the first or second baby had an average positive change (increase) in duration with the next one, whereas mothers who breastfed for a long time with the first or second baby

Table 2. Median and percentiles of breastfeeding duration with the first and second child. ${ }^{1}$

\begin{tabular}{lcc}
\hline & First child & Second child \\
\hline Sample size $(n)$ & 327 & 327 \\
Median & 4 & 5 \\
10th percentile & 2 & 2 \\
25th percentile & 3 & 3 \\
50th percentile & 4 & 5 \\
75th percentile & 8 & 9 \\
90th percentile & 12 & 14 \\
\hline
\end{tabular}

${ }^{1}$ All values other than sample size are in months. 
Table 3. Correlation coefficients between breastfeeding durations and duration changes.

\begin{tabular}{|c|c|c|c|c|c|}
\hline & $\begin{array}{c}\text { Breastfeeding } \\
1\end{array}$ & $\begin{array}{c}\text { Breastfeeding } \\
2\end{array}$ & $\begin{array}{c}\text { Breastfeeding } \\
3\end{array}$ & $\begin{array}{l}\text { Breastfeeding } \\
\text { change } 1-2\end{array}$ & $\begin{array}{c}\text { Breastfeeding } \\
\text { change } 2-3\end{array}$ \\
\hline Breastfeeding 1 & 1.00 & $\mathbf{0 . 6 3 9} * *$ & $0.479 * *$ & $-0.29 * *$ & -0.023 \\
\hline Breastfeeding 2 & & 1.00 & $0.494 * *$ & $0.552 * *$ & $-\mathbf{0 . 3 7} * *$ \\
\hline Breastfeeding 3 & & & 1.00 & -0.02 & $0.627 * *$ \\
\hline Breastfeeding change $1-2$ & & & & 1.00 & $-\mathbf{0 . 3 7} * *$ \\
\hline
\end{tabular}

Significant correlations are marked by $* *$ and set in bold; all are $p<0.01$.

had an average negative change (decrease) with the next. This relationship suggests a more complicated mechanism for regulating the duration of breastfeeding than was previously supposed. Fig. 1 shows a scatter plot diagram of breastfeeding duration with the second child ( $\mathrm{Y}$ axis) depending on the breastfeeding duration of the first child ( $\mathrm{X}$ axis). This graph illustrates the nature of the negative correlation described above. The turn point of the curve is at $8 \mathrm{mo}$, indicating that mothers who breastfed for 7 or fewer months and those who breastfed for 8 or more differed in their change in breastfeeding duration at the subsequent birth. To better specify this relationship, we performed a linear regression analysis, which also shows a negative relationship between first breastfeeding and breastfeeding change (second-first breastfeeding, beta $=-0.29, t=-5.44$, $p<0.001$ ), but was not related to maternal age at the first pregnancy, or to gestational age, gender or weight of the newborn. Fig. 2 illustrates the turn point at 8 mo.

Duration of the second breastfeeding was related to

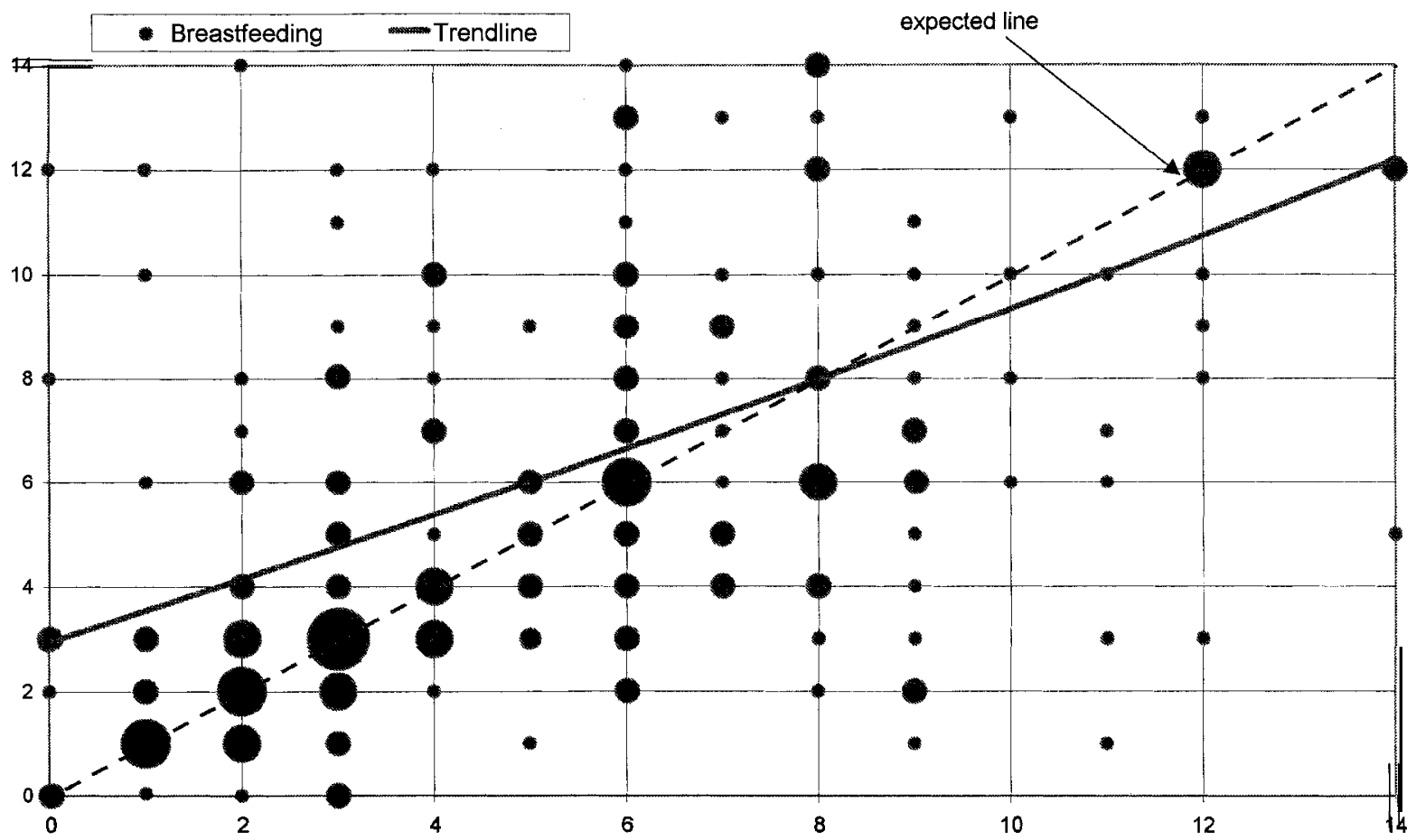

Fig. 1. Breastfeeding duration of the second child as a function of breastfeeding duration of the first child.

Title of the $\mathrm{X}$ axis: Breastfeeding the first child/months

Title of the Y axis: Breastfeeding the second child/months

Note: Fig. 1 depicts duration s of breastfeeding between 0 and 14 mo. Not shown on the figure are a small number of outliers $(n=22)$ with breastfeeding durations ranging from 15 to $42 \mathrm{mo}$ at the first breastfeeding; however, the trendline shown was plotted using all data.

- represents 1 case

- represents numbers of cases between 2 and 5;

represents numbers of cases between 6 and 10;

represents numbers of cases between 11 and 15, 


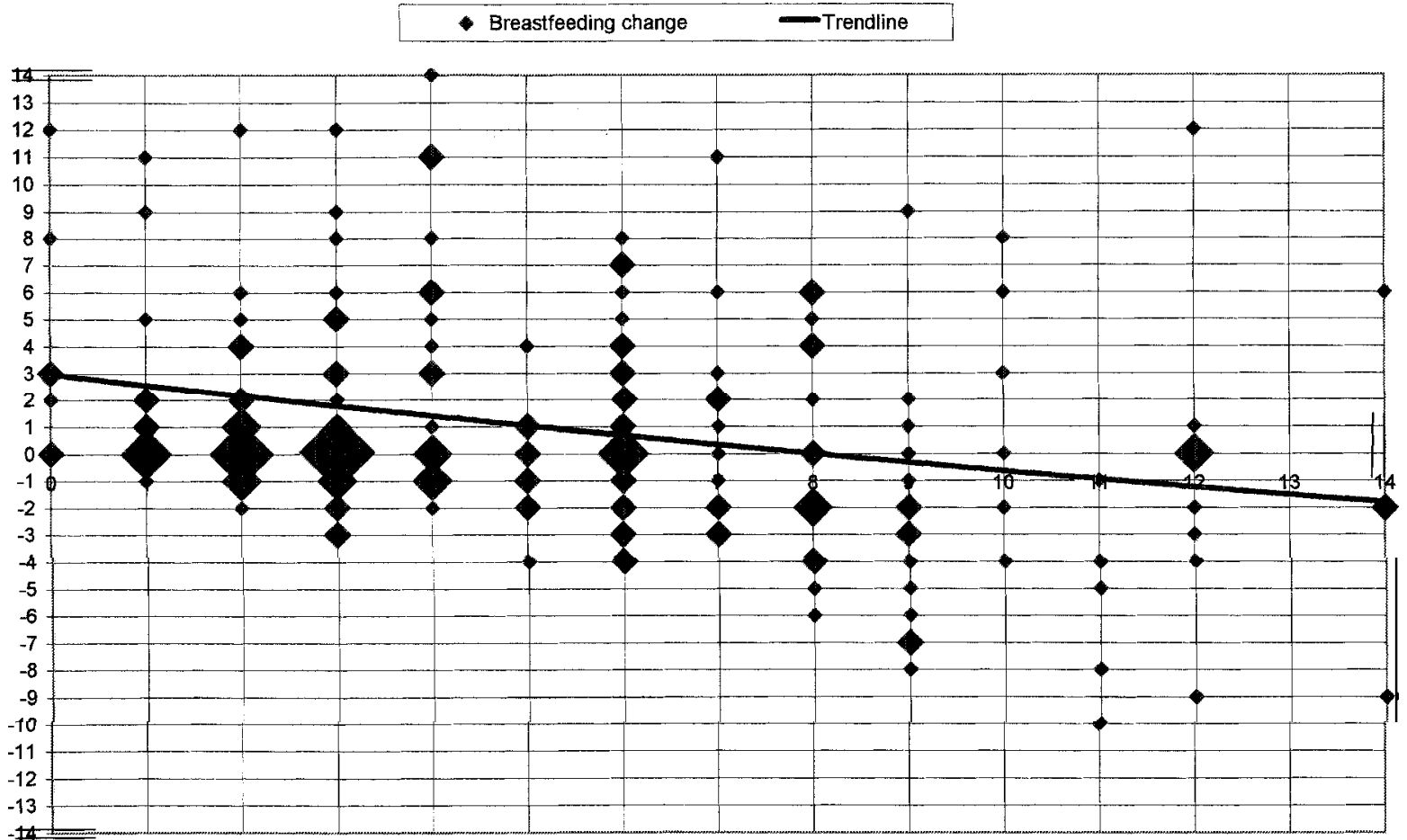

Fig. 2. Changes in breastfeeding duration as a function of breastfeeding duration of the first child.

Title of the $\mathrm{X}$ axis: Breastfeeding the first child/months

Title of the Y axis: Breastfeeding changes (second-first)/months

Note: Fig. 2 depicts duration of first breastfeeding s and breastfeeding changes between 0 and 14 mo. Not shown on the figure are a small number of outliers $(n=22)$ with breastfeedin g durations ranging from 15 to 42 mo at the first breastfeeding; however, the trendline shown was plotted using all data.

$\checkmark$ represents 1 case;

represents numbers of cases between 2 and 5;

$\checkmark$

represent $\mathrm{s}$ numbers of cases between 6 and 10;

represents numbers of cases between 11 and 15;

represents numbers of cases between 16 and 20;

represents 34 cases

the changes in breastfeeding between the second and third pregnancies (beta $=-0.37, t=-3.49, p=0.001$ ), and to the weight of the newborn (beta $=-0.23$, $t=-2.17, p=0.033$ ). However, it was not related to maternal age, gestational age, or the gender of the baby at the second pregnancy. Duration of the third breastfeeding likewise was unrelated to weight of the newborn, to gestational age of the newborn, maternal age, or to the gender of the baby at the third pregnancy.

\section{Subgroups in the sample}

In order to explore the above finding further, Wilcoxon paired comparison tests were used to separate the previously identified subgroups of mothers who breastfed for 7 or fewer months, or 8 mo or longer. Fig. 3 shows that mothers who breastfed for 7 or fewer months with their first baby significantly increased breastfeeding duration with the second baby $(n=242$, first baby mean $3.53 \mathrm{mo}, \mathrm{SD}=1.87$; second baby 5.31 mo, $\mathrm{SD}=5.18 ; \quad Z=-5.79, \quad p<0.0001)$, whereas mothers who breastfed their first baby for 8 or more months decreased their breastfeeding duration with the second one $(n=85$, first baby mean $13.11 \mathrm{mo}, \mathrm{SD}=7.1$, second baby mean $11.21 \mathrm{mo}, \mathrm{SD}=8.19, \mathrm{Z}=-2.99$, $p=0.03$ ).

Mothers who breastfed their second baby for 7 or fewer months significantly increased breastfeeding 


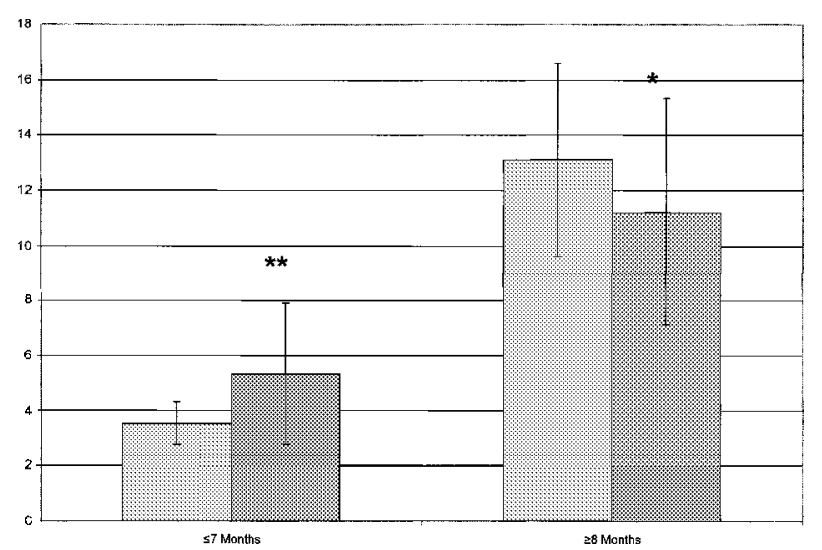

Fig. 3. Breastfeeding duration of the first and second child, with mothers grouped according to the first duration.

Title of the $X$ axis: $<7$ mo, $>8$ mo

Title of the Y axis: Breastfeeding duration /months

Footer: $* p<0.05, * * p<0.001$

duration with the third baby $(n=66$, second baby mean $3.39 \mathrm{mo}, \mathrm{SD}=1.78$; third baby mean $5.68, \mathrm{SD}=6.69$, $Z=-3.38, p=0.001$ ), whereas mothers who breastfed their second baby for 8 or more months tended to decrease breastfeeding duration with the third baby ( $n=23$, second baby mean $13.61, \mathrm{SD}=7.77$; third baby mean 11.39, $\mathrm{SD}=7.57, Z=-1.83, p=0.068$ ).

Thus, overall, the duration of breastfeeding at later births was positively related to duration at earlier births. However, mothers who breastfed for a relatively long time ( $\geq 8$ months) tended to decrease the duration of subsequent breastfeedings, even though their average duration was greater than that of mothers who had breastfed for shorter durations at their earlier births. This pattern of results, illustrated in Fig. 1, suggests that mothers whose duration of breastfeeding was at the extremes of the sample's distribution regressed toward the mean with subsequent births.

\section{Discussion}

Our results show that duration of breastfeeding increased with the second child, but did not significantly increase with the third. The mean breastfeeding duration in this Hungarian sample was 6.02 mo with the first child and 6.85 mo with the second. These figures are generally lower than those of some other countries. For example, Vestermark et al. (6) found a 7.9 mo average breastfeeding duration in their sample in Denmark. Average breastfeeding duration in Saudi Arabia was found to be relatively high: $11 \mathrm{mo}$ in urban and $13 \mathrm{mo}$ in rural communities (12). In the Latin American and Caribbean regions, average breastfeeding duration was found to be 11.8, and in Africa 19.3 mo (13).

While individual social learning may play a significant role, it is also important to consider possible effects of societal and cultural changes on patterns of breastfeeding. During the relatively long period included in this retrospective study, the incidence of breastfeeding increased in Hungary (Health and Social Statistical Yearbook; 14). The proportion of infants who were breastfed (only breastfed and breastfed with supplement) in their first 4 mo of life increased. In $1980,83.8 \%$; in $1985,87.8 \%$; in $1990,89.0 \%$; and in $1995,90.5 \%$ of Hungarian infants were breastfed. These prevalence data are relatively high: higher than those reported for Italy (15) and for Sweden, where prevalence of breastfeeding has increased to a high level in recent decades (16). The trend in changes of prevalence data in Hungary shows the same pattern as that reported for Sweden. Although these population data are not on breastfeeding duration, they tend to support the idea that a societal trend toward increased breastfeeding has been present in Hungary during this period. However, it is not possible to differentiate effects of societal change from those of individual learning, based on these data alone.

The second main result of this study was that mothers who breastfed their previous children for 7 or fewer months significantly increased breastfeeding duration with the subsequent child, while those who breastfed their infant for 8 or more months significantly decreased it. This apparent regression to the mean is an interesting phenomenon which may serve to optimize the duration of breastfeeding for the mother. Victora et al. (11) found a similar result in their sample of mothers from southern Brazil, suggesting that this phenomenon may be widespread. Although breastfeeding duration increased overall in this sample during a period of time when breastfeeding was gaining greater acceptance in Hungary, suggesting that societal changes may play a role, this finding is difficult to explain purely as a result of societal changes in breastfeeding.

Dugdale (17) hypothesized that in the evolution of human breastfeeding the mother-infant dyad develops an optimal length of breastfeeding, for the individual and also for the population. This optimal duration may serve to maximize survival for both infant and mother. Optimal duration for a mother may be regulated by factors such as food availability or the contraceptive effect of breastfeeding. In modern societies it may vary depending on the family's structure and a balance between optimal attachment in the mother-infant dyad and the mother's motives and needs. More recently, breastfeeding promotion programmes have played a role in certain societies, including Hungary. Another possible influence on the duration of breastfeeding is that in Western societies mothers may receive special encouragement and support to breastfeed in the first 6 mo, whereas this encouragement and support may decrease toward the end of the infant's first year of life.

It is intriguing to speculate that persistence of this kind of regulation mechanism in our societies provides an opportunity for breastfeeding promotion programmes to help mothers achieve an optimal duration 
of breastfeeding by influencing what mothers learn during the breastfeeding experience.

Acknowledgements.-Supported by grant OTKA F0 023194 from the Hungarian National Research Foundation. The authors are grateful to the reviewers for their help in revising this paper.

\section{References}

1. Suomi SJ, Ripp C. A history of motherless mother monkey mothering at the University of Wisconsin Primate Laboratory. In: Reite M, Caine N, editors. Child abuse: the nonhuman primate data. New York: Alan R. Liss; 1983. p. 49-78

2. Ryan AS, Martinez GA. Breast-feeding and the working mother: a profile. Pediatrics 1989; 83: 525-31

3. Macaulay AC, Hanusaik N, Beauvais JE. Breastfeeding in the Mohawk Community of Kahnawake: revisited and redefined. Can J Public Health 1989; 80: 177-81

4. Hill P, Humenick SS, Argubright TM, Aldag JC. Effects of parity and weaning practices on breastfeeding duration. Public Health Nurs 1997; 14: 227-34

5. MacGowan RJ, MacGowan CA, Serdula MK, Lane MJ, Joesoef RM, Cook FH. Breast-feeding among woman attending women, infants, and children clinics in Georgia, 1987. Pediatrics 1991; 87: $361-6$

6. Vestermark V, Hogdall CK, Plenov G, Birch M, Toftager-Larse n $\mathrm{K}$. The duration of breast-feeding. A longitudinal prospective study in Denmark. Scand J Soc Med 1991; 19: 105-9

7. Da Vanzo J, Starbird E, Leibowitz A. Do women's breastfeeding experience s with their first-born s affect whether they breastfeed their subsequent children? Soc Biol 1990; 37: 223-32
8. Becerra JE, Smith JC. Breastfeeding patterns in Puerto Rico. Am J Public Health 1990; 80: 695-7

9. Hornell A, Aarts C, Kylberg E, Hofvander Y, Gebre-Medhin M. Breastfeeding patterns in exclusively breastfe d infants: a longitudinal prospective study in Uppsala, Sweden. Acta Paediatr 1999; 88: 203-11

10. Jones DA, West RR, Newcombe RG. Maternal characteristic s associated with the duration of breast-feeding. Midwifery 1986; 2: $141-6$

11. Victora CG, Huttly SR, Barros FC, Vaughan JP. Breast feeding duration in consecutive offspring: a prospective study from southern Brazil. Acta Paediatr 1992; 81: 12-4

12. Al-Shehri SN, Farag MK, Baldo MH, Al-Amzrou YY, Aziz KMS. Overview of breastfeeding patterns in Saudi Arabia. J Trop Pediatr 1995; 41: 38-44

13. Perez-Escamilla R. Breastfeeding in Africa and the Latin American and Caribbean Region: the potential role of urbanization. J Trop Pediatr 1994; 40:137-43

14. Health and Social Statistical Yearbook. (Egeszsegugyi es Szocialis Statisztikai Evkonyv). Budapest: KSH; 1998. In Hungarian

15. Riva E, Banderali G, Agostoni, C, Silano M, Raaelli G, Giovannini M. Factors associated with the duration of breastfeeding in Italy. Acta Paediatr 1999; 88: 411-5

16. Zetterstrom R. Breastfeeding and infant-mother interaction. Acta Paediatr 1999; Suppl 430: 1-6

17. Dugdale AE. Evolution and infant feeding. Lancet 1986; 1: 8482: 670-3

Received Sept. 23, 1999; revisions received March 3 and June 6, 2000; accepted Aug. 31, 2000 